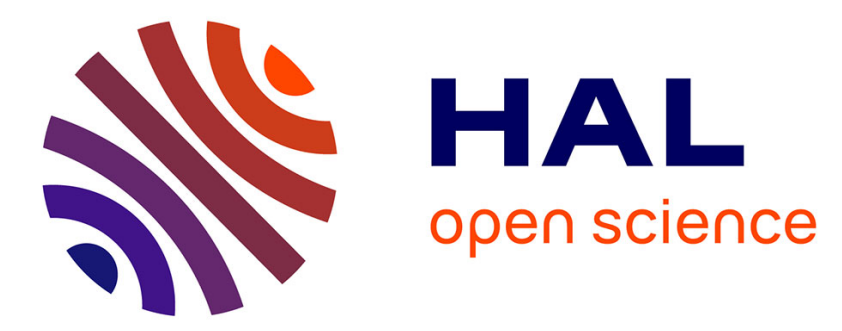

\title{
Utilisation d'ondes de Love pour déterminer l'épaisseur d'une couche de cuivre déposée électrolytiquement sur un substrat d'acier
}

\author{
P. Kielczyński, W. Pajewski
}

\section{- To cite this version:}

P. Kielczyński, W. Pajewski. Utilisation d'ondes de Love pour déterminer l'épaisseur d'une couche de cuivre déposée électrolytiquement sur un substrat d'acier. Revue de Physique Appliquée, 1986, 21 (9), pp.557-561. 10.1051/rphysap:01986002109055700 . jpa-00245473

\section{HAL Id: jpa-00245473 https://hal.science/jpa-00245473}

Submitted on 1 Jan 1986

HAL is a multi-disciplinary open access archive for the deposit and dissemination of scientific research documents, whether they are published or not. The documents may come from teaching and research institutions in France or abroad, or from public or private research centers.
L'archive ouverte pluridisciplinaire HAL, est destinée au dépôt et à la diffusion de documents scientifiques de niveau recherche, publiés ou non, émanant des établissements d'enseignement et de recherche français ou étrangers, des laboratoires publics ou privés. 


\title{
Utilisation d'ondes de Love pour déterminer l'épaisseur d'une couche de cuivre déposée électrolytiquement sur un substrat d'acier
}

\author{
P. Kielczyński et W. Pajewski \\ Laboratoire d'Acoustoélectronique, Institut des Problèmes Fondamentaux Techniques, \\ Académie Polonaise des Sciences, 00-049 Varsovie, ul. Świętokrzyska 21, Pologne
}

(Reçu le 20 mars 1986, révisé le 13 mai 1985, accepté le 15 mai 1986)

\begin{abstract}
Résumé. - Cet article présente les résultats d'une expérience ayant pour objet de déterminer l'épaisseur d'une couche superficielle d'un matériau élastique à l'aide d'ondes de surface transversales et horizontales du type de Love. Les principes théoriques de la méthode appliquée ont été développés par les auteurs précédemment (NDT Int. 18, $n^{\circ} 1$ (1985) 25-29). Les mesures ont été effectuées pour une couche de cuivre déposée par électrolyse sur un substrat d'acier. Plusieurs facteurs qui peuvent rendre la méthode considérée plus précise sont examinés.
\end{abstract}

\begin{abstract}
This paper presents experimental results of verification of the validity of the method used for determining the thickness of surface layers in elastic materials by means of shear horizontal surface waves of the Love type. Theoretical principles of the method were developed by the authors in a previous paper (NDT Inter. 18, no. 1 (1985) 25-29). The measurements have been carried out on steel samples covered with a copper layer. Some factors contributing to the accuracy of the method are given as well.
\end{abstract}

\section{Introduction.}

Jusqu'à présent, les structures superficielles de matériaux élastiques ont été étudiées surtout en utilisant les ondes de surface de Rayleigh [1]. Ces ondes possèdent, au minimum, deux composantes de déplacement mécanique, une composante longitudinale $(L)$ et une composante transversale verticale $(S V)$. Cependant, les ondes de Rayleigh ne sont ni longitudinales ni transversales. Elles peuvent être considérées comme une combinaison de ces composantes dépendant des paramètres élastiques du solide et des conditions aux limites à la surface de propagation. D'autre part, les ondes de Love, se propageant dans des structures superficielles stratifiées, ne possèdent qu'une seule composante transversale et horizontale de vibrations $(\mathrm{SH})$. A cause de l'existence d'une dimension caractéristique, par exemple l'épaisseur d'une couche superficielle, les ondes de Love sont toujours dispersives [2].

Dans le contrôle non destructif des matériaux l'avantage essentiel des ondes de Rayleigh est qu'elles existent potentiellement pour chaque sorte de structures superficielles d'un demi-espace élastique, indépendamment de la variation des paramètres des matériaux en fonction de la profondeur, c.-à-d. de la distance à la surface [3]. Les ondes de Love n'existent que dans des structures superficielles choisies [4] où la vitesse de phase de l'onde de volume $S H$ dans une couche superficielle est inférieure à celle dans le substrat.

Pour l'étude des structures superficielles l'utilisation des ondes de Love est théoriquement plus facile que celle des ondes de Rayleigh puisque la vitesse de phase des ondes de Love ne dépend que d'une constante élastique $c_{44}$. Par contre, la vitesse de phase d'ondes de Rayleigh dépend de deux constantes $c_{11}, c_{12}$, au minimum. Cela complique les expressions analytiques et également les procédures expérimentales ayant comme but l'analyse de structures superficielles [5].

En pratique, différents traitements de la surface des matériaux élastiques tels que l'électrolyse, la trempe, la nitruration, la boruration, l'implantation ou la diffusion ionique peuvent produire des couches superficielles où la vitesse de phase des ondes de volume SH peut être inférieure à celle du solide; ainsi les ondes de Love peuvent être utilisées dans le contrôle non-destructif des structures superficielles.

Dans la méthode expérimentale développée par les auteurs [6], l'épaisseur d'une couche superficielle est déterminée à partir de la courbe de dispersion de 
l'onde de Love/mode fondamental/ qui se propage dans la structure examinée. Au point de vue du formalisme mathématique, la méthode appliquée représente un problème spectral inverse de Sturm-Liouville [7].

Dans ce travail on présente les résultats préliminaires de vérification expérimentale confirmant la validité de la méthode élaborée. On a étudié l'épaisseur d'une couche de cuivre déposée par électrolyse sur un substrat d'acier.

\section{La méthode expérimentale.}

Les mesures de la vitesse de phase de l'onde superficielle de Love, se propageant dans la structure stratifiée cuivre-acier (Fig. 1), ont été effectuées à l'aide du dispositif expérimental Matec, en utilisant la méthode de réflexion développée par Papadakis [8]. Cette méthode est fondée sur la détermination du temps qui s'écoule entre deux échos successifs des impulsions de l'onde de surface réfléchie dans la structure limitée du guide superficiel (Fig. 1).

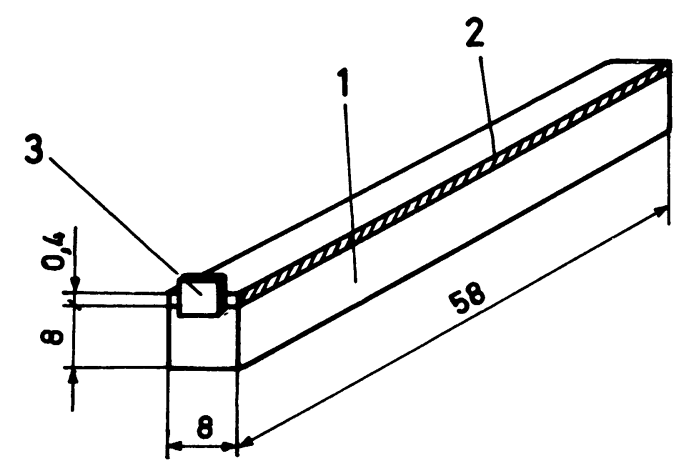

Fig. 1. - Structure stratifiée superficielle cuivre-acier étudiée à l'aide des ondes de surface transversales et horizontales de Love.

1. Echantillon d'acier, 2. couche de cuivre, 3. transducteur à plaque.

[Layered surface structure copper-steel examined by shear horizontal surface waves of the Love type.

1. Steel sample, 2. copper layer, 3. piezoelectric plate transducer.]

Les mesures ont porté sur des échantillons d'acier en forme de parallélépipède de dimensions $8 \times 8 \times$ $58 \mathrm{~mm}^{3}$, Sur une surface latérale de l'échantillon de dimensions $8 \times 58$ on a déposé par voie galvanique une couche de cuivre d'épaisseur $400,0 \pm 10 \mu \mathrm{m}$. Les échantillons étudiés ont été fabriqués avec précision pour que l'amplitude des impulsions de l'onde de surface diminue selon une fonction exponentielle. Les impulsions de l'onde de surface ont été excitées et détectées à l'aide de transducteurs piézoélectriques à plaque [9], disposés d'une manière convenable sur le chant du guide (Fig. 1). Ces transducteurs sont des céramiques piézoélectriques polarisées du type PZT et aussi des cristaux de niobate de lithium $\left(\mathrm{LiNbO}_{3}\right)$. Ils ont été excités par des impulsions électriques rectangulaires de durée 1- $\mu$ s avec une fréquence porteuse 1-10 MHz. La limite haute et la limite basse de la fréquence utilisée dépendaient de l'épaisseur de ces transducteurs. Pour des fréquences supérieures à $10 \mathrm{MHz}$, l'épaisseur d'un transducteur est trop petite pour qu'on puisse assurer sa résistance mécanique. Dans ce cas, on peut utiliser les vibrations harmoniques de transducteurs de fréquences supérieures à $10 \mathrm{MHz}$. D'autre part, pour des fréquences inférieures à $1 \mathrm{MHz}$, l'épaisseur d'un transducteur est alors trop grande en comparaison des autres dimensions de la plaque. Ce dernier effet provoque des oscillations parasites non souhaitables. Le contact mécanique entre les transducteurs à plaque et la structure stratifiée fut réalisé à l'aide d'une résine époxide possédant une grande viscosité.

Bien que l'ensemble du dispositif expérimental du type de Matec assurât une précision de mesure de l'ordre de $10^{-7}$, celle pour l'onde de Love n'était plus que d'environ $1 \%$, lorsque les échos successifs de l'onde de surface étaient perturbés par la forte dispersion de l'onde de Love se propageant dans la structure stratifiée cuivre-acier. Les échos successifs de l'onde de Love ont pu être superposés (sur l'écran d'un oscilloscope), suivant la méthode de Papadakis [8], avec une erreur de moins d'une demi longueur d'onde de fréquence porteuse.

Les figures $2 a$ et $2 b$ présentent des oscillogrammes typiques illustrant les impulsions de l'onde de Love réfléchie dans la structure examinée cuivre-acier. La surface de propagation était libre (Fig. 2a) ou bien était chargée par une couche mince de résine époxide très visqueuse (Fig. $2 \mathrm{~b}$ ).

Les résultats des mesures de la vitesse $v$ de l'onde de Love, en fonction de la fréquence $f$, sont présentes dans le tableau I. Pour les fréquences employées, $f=1,13$ $6,14 \mathrm{MHz}$, la longueur de l'onde de Love changeait de $2292 \mu \mathrm{m}$ à $341 \mu \mathrm{m}$ et était comparable à l'épaisseur mesurée de la couche de cuivre, $d=400 \mu \mathrm{m}$. Pour des fréquences supérieures à env. $6 \mathrm{MHz}$, on a pu observer des modes supérieurs de l'onde de Love dont la présence n'était pas souhaitée pendant les mesures de vitesse d'un mode fondamental. La vitesse de phase $v_{0}$ de l'onde de volume $S H$ se propageant dans le substrat d'acier a été mesurée à l'aide des mêmes transducteurs à plaque. Cette vitesse ne changea pas dans la bande de fréquence de 5 à $25 \mathrm{MHz}-v_{0}=$ $3207 \mathrm{~m} / \mathrm{s}$. A partir du tableau I on voit que pour des fréquences supérieures à environ $3,36 \mathrm{MHz}$ la vitesse de l'onde de Love est à peu près constante, c.-à-d. suivant la notation introduite par les auteurs dans [6], $v(0)=2097 \mathrm{~m} / \mathrm{s}$. D'autre part, pour la fréquence intermédiaire $f_{\mathrm{i}}=1,13 \mathrm{MHz}$, la vitesse de l'onde de Love est égale à $v\left(f_{\mathrm{i}}\right)=2590 \mathrm{~m} / \mathrm{s}$. Puisque la masse volumique de la couche de cuivre obtenue électrolytiquement est inférieure à celle du cuivre monocristallin on peut poser : $\rho_{1} \cong \rho_{0}=7,8 \times 10^{3} \mathrm{~kg} / \mathrm{m}^{3}$ [10] et [11], 

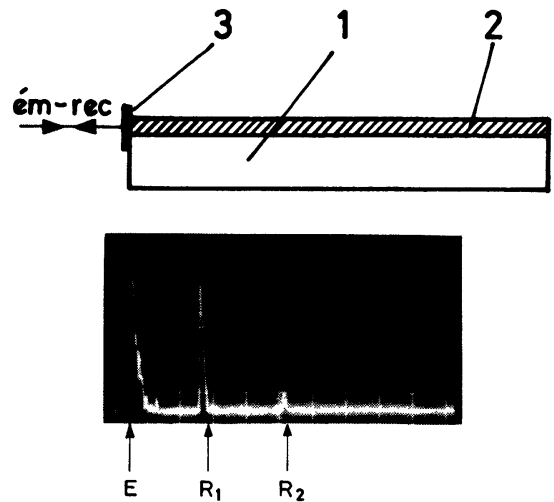

$X=20 \mu s / e ́ c h$ $Y=5 \mathrm{~V} / \mathrm{keh}$.
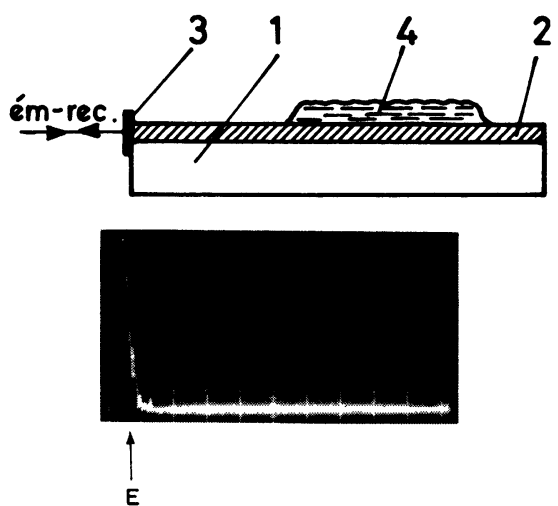

b)

Fig. 2. - Oscillogrammes typiques d'impulsions de l'onde de Love se propageant dans la structure stratifiée cuivreacier; a. surface de propagation libre; b. surface de propagation chargée d'une couche de résine.

1. Echantillon d'acier, 2. couche de cuivre, 3. transducteur piézoélectrique à plaque, 4 . couche de résine. $\mathrm{E}$ : impulsion électrique, $\mathbf{R}_{1}, \mathbf{R}_{\mathbf{2}}$ : deux échos de l'onde de Love. Fréquence porteuse d'impulsions $10 \mathrm{MHz}$.

[Typical oscillograms of impulses of the Love wave propagating in the layered structure copper-steel; a. free surface of propagation; b. surface of propagation loaded with a layer of resin.

1. Steel sample, 2. copper layer, 3. piezoelectric plate. transducer, 4. resin layer. $E$ : electrical impulse, $R_{1}, R_{2}$ : two echoes of the Love wave. Carrier frequency of the impulses $10 \mathrm{MHz}$.] ou $\rho_{0}, \rho_{1}$ denotent les masses volumiques d'un substrat d'acier et d'une couche de cuivre, respectivement.

3. Calcul de l'épaisseur de la couche de cuivre à partir de la courbe de dispersion de l'onde de Love.

Pour déterminer l'épaisseur d'une couche superficielle selon la méthode examinée, il suffit en principe de mesurer la vitesse de phase de l'onde de Love aux trois points : fréquence " basse », fréquence " haute » et fréquence intermédiaire [6]. Pour la fréquence « basse » ou « haute » la longueur de l'onde de Love doit être notablement plus grande ou plus petite que l'épaisseur de la couche examinée. Pour la fréquence intermédiaire, il est pratique de choisir la fréquence pour laquelle l'épaisseur étudiée est égale à environ la longueur de l'onde de volume $S H$, de même fréquence, se propageant dans le substrat. Un tel choix de fréquence intermédiaire conduit à des erreurs modérées de la méthode expérimentale considérée [6].

Le coefficient de flexibilité $s_{44}$ au voisinage de la surface libre de la couche superficielle, dénoté par $s_{0}+\Delta s$, et le coefficient dans la profondeur de l'échantillon, dénoté par $s_{0}$, sont calculés à partir des expressions élémentaires (Eq. (18) dans [6]) :

$$
\begin{gathered}
s_{0}+\Delta s=\rho_{1}^{-1}(v(0))^{-2} \\
s_{0}=\rho_{0}^{-1} v_{0}^{-2}
\end{gathered}
$$

ou $v_{0}$ et $v(0)$ sont les vitesses de phase de l'onde de volume plane $S H$ se propageant dans des milieux caractérisés par les coefficients de flexibilité $s_{0}$ et $s_{0}+\Delta s$, respectivement.

La valeur auxiliaire de $\alpha d$ est déterminée par les équations suivantes (Eqs. (A.5) et (17) dans [6]) :

$$
\begin{gathered}
\operatorname{erf}(\alpha d)-\frac{\alpha d}{(\pi)^{1 / 2}} \exp \left(-\alpha^{2} d^{2}\right)=\frac{\lambda}{c} \\
\frac{\lambda}{c}=\frac{s_{0}}{\Delta s}\left(\left(\frac{v_{0}}{v\left(f_{\mathrm{i}}\right)}\right)^{2}-1\right)
\end{gathered}
$$

Tableau I. - Valeurs expérimentales de vitesse de phase $v$ d'onde de Love se propageant dans la structure stratifiée cuivre-acier, en fonction de la fréquence $f$. $\lambda$-longueur de l'onde de Love.

[Phase velocity $v$ of Love wave propagating in the layered structure copper-steel as a function of frequency $f$. $\lambda$-wavelength of the Love wave.]

\begin{tabular}{|l|r|r|r|r|r|r|r|r|r|}
\hline \multicolumn{2}{|l|}{$f(\mathrm{MHz})$} & 1,13 & 2,5 & 3,18 & 3,46 & 4,12 & 4,71 & 5,36 & 6,14 \\
\hline$v(\mathrm{~m} / \mathrm{s})$ & 2590,0 & 2185,0 & 2114,0 & 2097,0 & 2097,0 & 2099,0 & 2098,0 & 2097,0 \\
\hline \multirow{2}{*}{ Erreur } & $\mathrm{m} / \mathrm{s}$ & $\pm 31,2$ & $\pm 26,5$ & $\pm 19,5$ & $+17,6$ & $\pm 14,8$ & $\pm 13,0$ & $\pm 11,4$ & $\pm 9,95$ \\
\cline { 2 - 10 } & $\%$ & 1,22 & 1,21 & 0,92 & 0,84 & 0,71 & 0,62 & 0,54 & 0,47 \\
\hline$\lambda(\mu \mathrm{m})$ & 2292,0 & 874,0 & 665,0 & 606,0 & 509,0 & 445,0 & 391,0 & 341,0 \\
\hline
\end{tabular}


où $v\left(f_{\mathrm{i}}\right)$ est la vitesse de l'onde de Love de fréquence intermédiaire $f_{\mathrm{i}}$ et $\alpha$ un coefficient numérique résultant de la méthode variationnelle considérée [6].

L'épaisseur étudiée de la couche de cuivre $d$ est déterminée par l'équation suivante (Eq. (A.6) dans [6]) :

$$
d=\frac{\lambda_{0}}{2 \pi}\left(\frac{s_{0}}{\Delta s}\right)^{1 / 2}\left(\frac{\alpha d(\pi)^{1 / 2}}{2}\right)^{1 / 2} \exp (\alpha d)
$$

où $\lambda_{0}=v_{0} / f_{\mathrm{i}}$ et la valeur de $\alpha d$ est donnée par l'équation (2).

Les valeurs de $v_{0}, v(0), v\left(f_{\mathrm{i}}\right)$ sont évaluées expérimentalement :

$$
\begin{aligned}
v_{0} & =3207,0 \pm 28 \mathrm{~m} / \mathrm{s} \\
v(0) & =2097,0 \pm 10 \mathrm{~m} / \mathrm{s} \\
v\left(f_{\mathrm{i}}\right) & =2590,0 \pm 31 \mathrm{~m} / \mathrm{s}
\end{aligned}
$$

En reportant les valeurs mesurées $v_{0}, v(0), v\left(f_{\mathrm{i}}\right)$ et $f_{\mathrm{i}}$ dans les équations (1) à (2a), on obtient

$$
\begin{aligned}
s_{0} / \Delta s & =0,76914 \\
\alpha d & =0,645 \\
\operatorname{erf}(\alpha d) & =0,6383 \\
\lambda / c & =0,3982 \\
\lambda_{0} & =2838,0 \mu \mathrm{m} .
\end{aligned}
$$

Finalement, l'épaisseur cherchée de la couche superficielle de cuivre calculée à partir de l'équation (3) est égale à : $d=562,5 \mu \mathrm{m}$.

\section{Discussion et conclusions.}

Les raisons de la divergence entre la valeur expérimentale $d=562,5 \mu \mathrm{m}$ et la valeur réelle $d=400,0 \mu \mathrm{m}$ de l'épaisseur de la couche de cuivre sont plus apparentes après examen des courbes de dispersion (vitesse de phase) pour le mode fondamental de l'onde de Love qui se propage dans la structure stratifiée cuivreacier(Fig. 3). Les courbes ont été tracées pour plusieurs valeurs de l'épaisseur de la couche de cuivre, $d=$ 200,0-1 000,0 $\mu \mathrm{m}$ [12]. Les valeurs expérimentales sont aussi portées sur la figure 3 . Les points expérimentaux ( I ) sont situés généralement en dessous de la courbe de dispersion correspondante à l'épaisseur $d=400,0 \mu \mathrm{m}$. De plus, on peut constater qu'ils sont plus proches de la courbe de dispersion de la vitesse de groupe [13] pour la même épaisseur de la couche superficielle. Il est probable que cet effet est dû à la grande dispersion de l'onde de Love se propageant dans la structure cuivre-acier où par exemple $v_{0}=$ $3207,0 \mathrm{~m} / \mathrm{s}$ et $v(0)=2097,0 \mathrm{~m} / \mathrm{s}$. En outre, en utilisant la méthode de Papadakis [8] on détermine de facto la vitesse de groupe, pas la vitesse de phase, puisqu'on mesure le temps entre les maxima des trains de l'onde, se propageant dans la structure fortement dispersive [14].

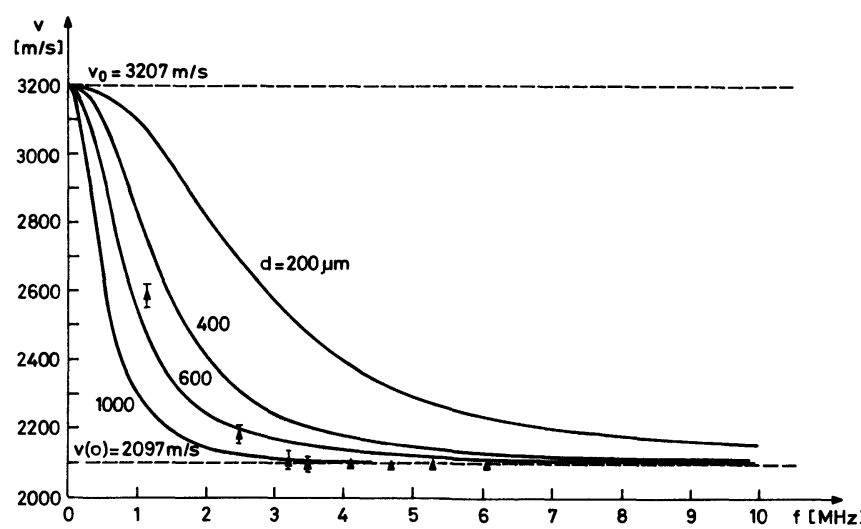

Fig. 3. - Vitesses de phase $v$ de l'onde de Love se propageant dans la structure stratifiée cuivre-acier calculées en fonction de la fréquence $f$, pour plusieurs valeurs de l'épaisseur d'une couche de cuivre $d$.

(I) : points expérimentaux obtenus à l'aide de la méthode de Papadakis.

[Calculated phase velocities $v$ of the Love wave propagating in the layered structure copper-steel versus frequency $f$, for different values of the copper layer $d$.

(I) : experimental points obtained via the Papadakis method.]

En tenant compte des résultats expérimentaux et numériques présentés dans ce travail on peut conclure :

1) L'épaisseur de la couche de cuivre calculée à partir de la courbe de dispersion de l'onde de Love, $d=562,5 \mu \mathrm{m}$, est supérieure d'environ $40 \%$ à la valeur réelle, $d=400,0 \mu \mathrm{m}$. Cette différence peut être attribuée à la relativement grande dispersion de l'onde de Love se propageant dans la structure stratifiée cuivre-acier.

2) En utilisant la méthode d'impulsion de Papadakis on ne peut déterminer en principe que la vitesse de groupe. Il est à noter que dans des structures faiblement dispersives la vitesse de phase et la vitesse de groupe sont presque les mêmes, par exemple dans les structures superficielles produites par la trempe chimique ou thermique. Il serait utile de développer des méthodes à l'aide desquelles on pourrait déterminer l'épaisseur de la couche superficielle à partir de la courbe de dispersion de la vitesse de groupe, au lieu de la méthode présentée dans cet article où on utilise une courbe de dispersion de la vitesse de phase. On peut aussi développer des méthodes de mesure de vitesse de phase pour des ondes qui se propagent dans des solides dispersifs [14-16].

3) Il est possible, en principe, d'exciter et de détecter les ondes de surface de Love à l'aide de transducteurs piézoélectriques à plaque seulement dans des conditions de laboratoire lorsque les échantillons examinés sont assez longs et soigneusement préparés. En pratique industrielle ces conditions peuvent être difficiles à réaliser. Pour cette raison le développement de nouvelles méthodes de génération et de détection d'ondes de surface est un problème actuel [17]. 


\section{Bibliographie}

[1] Rousseau, J., Pouliquen, J., Defebvre, A., Vitesse de propagation et localisation de l'énergie d'ondes acoustiques de surface dans des milieux stratifiés, Revue Acoustique 56 (1981) 11-15.

[2] KiełcZYŃski, P., Propagation of surface SH waves in nonhomogeneous media, J. Tech. Phys. (Warsaw) 1 (1981) 73-78.

[3] Barnett, D. M., Lothe, J., Consideration of the existence of surface wave (Rayleigh) solutions in anisotropic elastic crystals, J. Phys. F 4 (1974) 671-686.

[4] AChenbach, J. D., Wave Propagation in Elastic Solids (North-Holland, Amsterdam) 1973.

[5] Wasilewski, A., Etude de Couches Superficielles d'un Solide à l'Aide de l'Onde de Rayleigh. 1980. Thèse Doctorat ès Sciences, Institut des Problèmes Fondamentaux Techniques, Académie Polonaise des Sciences, 00-049 Varsovie, ul. Świetokrzyska 21, Pologne (en polon.).

[6] KiełczyŃski, P., PAJewski, W., Determination of the depth of a non-homogeneous surface layers in elastic materials using shear surface waves, NDT Inter. 18, no 1 (1985) 25-29.

[7] Baltes, H. P., ed., Inverse Source Problems in Optics, ch. 3 (Springer, Berlin) 1978.

[8] Papadakis, E. P., Ultrasonic phase velocity measurements by the pulse-echo-overlap method incorporating diffraction phase corrections, J. Acoust. Soc. Am. 42, no 5 (1967) 1045.

[9] Nguyen Viet KInH, Pajewski, W., Generation of acousto-electrical waves using a source of trans- verse vibrations, Arch. Acoust. (Warsaw) 5, no 3 (1980) 261-274.

[10] Kittel, C., Introduction to Solid State Physics, ch. 1 (Wiley, New York) 1966.

[11] Obraz, J., Ondes Ultrasonores dans la Technique des Mesures (WNT Varsovie) 1983 (en polonais).

[12] Farnell, G. W., Properties of elastic surface waves, in Physical Acoustics, vol. VI, ed. by W. P. Mason and R. N. Thurston (Academic, New York) 1970, p. 109-166.

[13] MikLowitz, J., The Theory of Elastic Waves and Waveguides (North-Holland, Amsterdam) 1978.

[14] Lynworth, L. C., Rea, W. R., Continuous wave transmission techniques for measuring ultrasonic phase and group velocities in dispersive materials and composites, J. Acoust. Soc. Am. 70, no 6 (1981) 1699-1703.

[15] SACHSE, W., YIH-Hsing PAO, On the determination of phase and group velocities of dispersive waves in solids, J. Appl. Phys. 49, no 8 (1978) 4320-4327.

[16] Papadakis, E. P., Ultrasonic velocity and attenuation : measurement methods with scientific applications, in Physical Acoustics, vol. XII, ed. W. P. Mason and R. N. Thurston (Academic, New York) 1976, 277-374.

[17] Kotlicka, E., Latuszek, A., Nouvelle méthode d'excitation d'ondes de surface ultrasonores à la surface de solides arbitraires, Proceedings of the XXXII Open Seminar on Acoustics. OSA 1985, Cracovie, September 1985, 255-257 (en polonais). 